Al Khateeb, Ayman :

Optimierung von Fließproduktionssystemen mit Nachbearbeitungsschleifen

Zuerst erschienen in:

Wissenschaftliche Gesellschaft für Technische Logistik :

Tagungsband // 6. Fachkolloquium der Wissenschaftlichen Gesellschaft für Technische Logistik (WGTL) . - Garbsen : PZH Produktionstechnisches Zentrum, ISBN 978-3-941416-71-0. - 2010, S. $277-288$ 


\title{
Optimierung von Fließproduktionssystemen mit Nachbearbeitungsschleifen
}

\author{
Dipl.-Ing. Ayman Al Khateeb \\ Technische Universität IImenau \\ Institut für rechnerunterstützte Produktion \\ Fachgebiet Fabrikbetrieb
}

Abstract: Die Verteilung der Arbeitsmenge auf die Bearbeitungsstationen in einer Fließproduktionslinie (FPL) ist im Prinzip möglichst gleichmäßig durch Fließbandabstimmung durchzuführen. Im Fall nichtlinearer Fließproduktionssysteme (FPS) führen die Ein- und/oder Ausschleusungen zur Änderung der Stationsauslastungen und dadurch zur Entstehung von Engpässen bzw. Verlustzeiten.

Der Beitrag beschreibt eine neue Strategie zur verbesserten Verteilung der Arbeitsmenge in nichtlinearen FPS. Als Beispiel solcher Systeme wird ein FPS mit Nachbearbeitungsschleife (NBS) behandelt. In Analogie zu hydraulischen Anlagen, in denen eine Beziehung zwischen den Werten der Querschnittsflächen von Rohren und dem Volumenstrom besteht, können die Bearbeitungszeiten der Stationen in den Fließlinien vor und nach den Vernetzungselementen (Einschleusung- und Zuführungselemente) bestimmt werden. Anschließend wird die Beziehung zwischen diesen Bearbeitungszeiten analysiert und in Formeln übersetzt. Weiterhin wird ein Algorithmus zur Durchführung der Optimierung entwickelt und angewandt. Durch eine Wiederholung der Arbeitsverteilung auf die Stationen wird bei diesem Vorgehen der Engpass des betrachteten Systems vermieden.

\section{$1 \quad$ Einleitung und Problemstellung}

Ein FPS setzt sich in der Regel aus Bearbeitungsstationen (Fertigungs- oder Montagestationen, im Folgenden auch einfach bezeichnet als „Stationen“) zusammen, die miteinander durch entsprechende Transportmittel und/oder Puffer verbunden sind. Je nach der Verbindungsform unterscheidet man zwischen linearen und nichtlinearen FPS.

Zur Abstimmung von Stationsleistungen in linearen FPS bietet die Literatur sehr viele Forschungsarbeiten an, wie z.B. [Sch99], [Boy05]. Wird ein Vernetzungselement einer FPL hinzugefügt, ist die Abstimmung der Bearbeitungszeiten der Stationen erneut durchzuführen, um Leistungsverluste möglichst zu vermeiden. Zu dieser Fragestellung findet man nur sehr wenige Hilfestellungen in der Fachliteratur.

Um die Untersuchung der Bearbeitungszeiten auf beiden Seiten eines Vernetzungselements in einem FPS durchzuführen, wird ein praxisnahes Modell gesucht, das ein Verzweigungs- und ein Zuführungselement einschließt. Das Modell ist ein FPS mit NBS. 


\subsection{Qualitätskontrolle und NBS}

Da die Bearbeitungsprozesse an den Stationen in einer FPL nicht immer perfekt sein können, ist die Qualitätskontrolle (QK) den Produktionsprozessen hinzuzufügen. Wobei eine Verzweigung nach der QK auszulegen ist, um die mangelhaften Produkte auszusondern oder nachzuarbeiten und zur Hauptlinie zurückzugeben. Dadurch ist eine Zusammenführung vor der QK einzurichten. Es entsteht zusätzliche Arbeit für die Stationen in der Hauptlinie, indem nachbearbeite Erzeugnisse erneut zur Bearbeitung vorliegen. Ein Engpass wird verursacht, der zur Veränderung bzw. zur Verringerung des Gesamtdurchsatzes führt.

In der Literatur finden sich wenige Referenzen, die sich mit nichtlinearen FPS beschäftigen wie z.B. [Ger91], [Hel00]. NBS wurde gelegentlich in der Literatur behandelt (vgl. [Li04], [Hel99] und [HM03]). Die Abbildung 1 stellt eine der häufig in der Literatur untersuchten Strukturen dar.

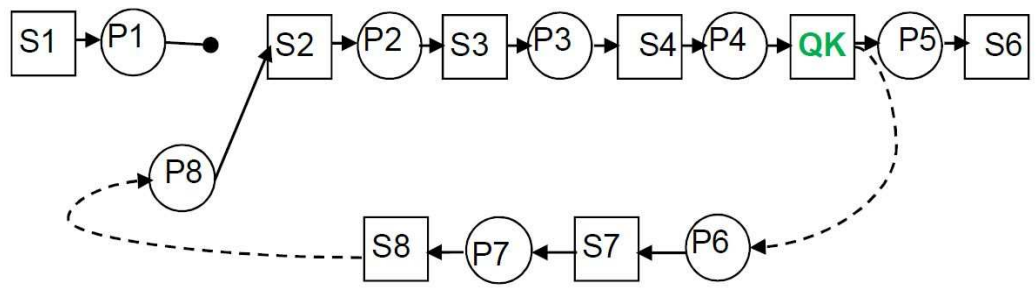

Abbildung 1: Struktur einer Nachbearbeitungsschleife

\subsection{Funktion der NBS}

Die QK überprüft die Erzeugnisse und gliedert sie normalerweise in drei Typen:

- Perfekte Erzeugnisse werden zu der Nachfolgerstation in der Hauptlinie weitergegeben.

- Fehlerhafte Erzeugnisse müssen in der Nebenlinie weiterlaufen. In den Stationen S7 bzw. S8 (siehe Abbildung 1) werden sie nachbearbeitet und zurück zur Station S2 in der Hauptlinie transportiert. Sie werden genauso wie die von der Station S1 kommenden Erzeugnisse behandelt und in den Stationen S2, S3 und S4 noch einmal bearbeitet. Das bedeutet, diese Erzeugnisse können ein weiteres Mal fehlerhaft bearbeitet werden.

- Ausschuss muss aus dem System entfernt werden.

Die Qualitätskontrolle ist unmittelbar nach der Station vorzusehen, in der die möglicherweise fehlerhaften Erzeugnisse produziert werden, um auf diese Weise die Anzahl der Stationen in der NBS zu minimieren. Sollten in einem FPS mehrere Stationen hintereinander liegen, die fehlerhafte Erzeugnisse produzieren könnten, sollte die QK aus demselben Grund nach der letzten dieser Stationen eingesetzt werden (vgl. [Hel99]). Die Ausschleusungsvorgänge durch die Qualitätskontrolle haben in Bezug auf die Nachfolgerstationen zur Folge, dass sich deren Arbeitsmenge reduziert. 


\section{Ziele und Vorgehensweise dieser Arbeit}

Die Berechnung der Bearbeitungszeiten von Fließlinien auf beiden Seiten der Vernetzungselemente basiert auf den Annahmen der idealen Situation. Dabei sollten keine Zeit- bzw. Leistungsverluste an den Stationen auftreten. Die Transportzeiten zwischen Stationen und die Zeitverluste in den Vernetzungselementen wären vernachlässigbar. Um Verschwendungen zu vermeiden sollte das vorliegende FPS mit dem Ziel optimiert werden, dieser idealen Situation möglichst nahe zu kommen oder sie gar zu erreichen.

Um die Methode der Optimierung zu vereinfachen, wird ein Vergleich zwischen hydraulischen Rohren zum Transportieren von flüssigen (inkompressiblen) Medien und den Stationen innerhalb eines FPS zur Herstellung von Erzeugnissen vorgenommen. In der Folge werden Formeln abgeleitet, die die Beziehung zwischen den Bearbeitungszeiten der Stationen vor und nach den Vernetzungselementen darstellen. Anschließend wird ein Algorithmus entwickelt, der die Optimierungsschritte im Allgemeinen interpretiert.

Anhand eines Beispiels wird anschließend die Verbesserungsmöglichkeiten durchgeführt und die optimierte Struktur dargestellt.

\subsection{Hydraulische Analogie}

Häufig werden Strukturen, die der in Abbildung 1 ähneln, unter der Annahme so behandelt, dass die mittleren Bearbeitungszeiten von Stationen in Haupt- und Nebenlinie gleich sind (vgl. [Hel99]). Um diese Annahme zu diskutieren, wird ein Vergleich zwischen hydraulischen Rohren und FPS durchgeführt.

Man gehe im Folgenden also davon aus, dass die diskreten Erzeugnisse wie Flüssigkeit durch ein Rohr fließen und die Stationen als Ventile und/oder Pumpen ${ }^{1}$ zur Förderung des Mediums zur nächsten Station dienen. Gemäß dieser Vorstellung werden die Erzeugnisse kontinuierlich an den Stationen bearbeitet (vgl. [HM03]).

Unter Bezugnahme auf Abbildung 2 und unter der Annahme, dass die Erzeugnisse ${ }^{2}$ im Volumen $\mathrm{V}$ verschmelzen, kann man die Formeln des Volumenstroms $Q$ in Flüssigkeiten benutzen:

$\mathrm{Q}=\frac{\mathrm{v}}{\mathrm{t}} ; \mathrm{t}$ : Zeitdauer der Untersuchung (Strömung)

$\mathrm{Q}=\mathrm{c} \cdot \mathrm{A}$; c: mittlere Strömungsgeschwindigkeit, A: Querschnittsfläche (QSF) des Rohrs

Ist c in verschiedenen Rohren konstant, sind die QSF der Rohre proportional mit dem Volumenstrom auszulegen.

Die Abbildung 2.b zeigt, dass der gesamte Volumenstrom $Q$ für die Stationen der Anzahl j die Summe der einzelnen Volumenströme Q1 und Q2 ist. Das bedeutet, die QSF A ist gleich der Summe aus A1 und A2.

\footnotetext{
${ }^{1}$ In der Abb. 2 werden nur zwei Stationen als Ventile und Pumpen gezeigt. Sie liegen an den Grenzen der NBS.

${ }^{2}$ Ausgehend von gleichem Volumen der Erzeugnisse.
} 
(a) untersuchte Struktur

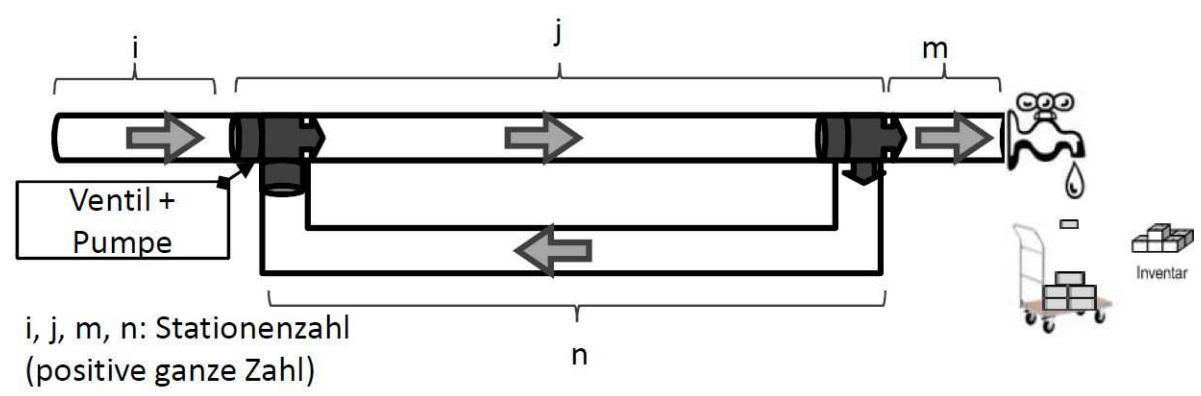

(b) optimierte Struktur

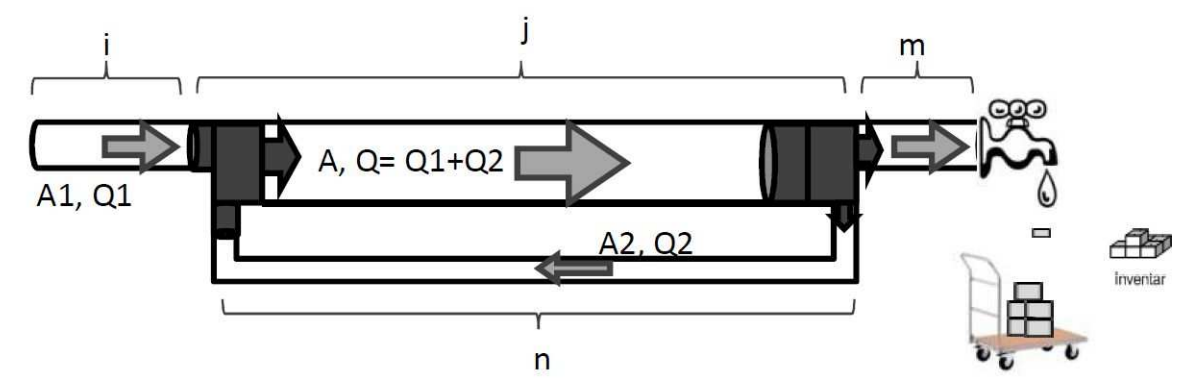

Abbildung 2: Darstellung der FPL als Rohrleitung und Stationen als Ventile

Entsprechend ist erkennbar, dass eine Beziehung zwischen den Bearbeitungszeiten der Stationen vor und nach den Vernetzungselementen besteht. Infolgedessen ist der Begriff „Bearbeitungszeit“ der Station in der FPL darzustellen.

\subsection{Arbeitsverteilung}

Die erforderliche Bearbeitungszeit zur Herstellung eines Produkts in einer FPL setzt sich aus einzelnen Arbeitselementen zusammen. Aus ökonomischen oder technischen Gründen kann unterstellt werden, dass diese nicht mehr unterteilt werden können (vgl. [KH04]). Die Abbildung 3 zeigt einen Vorranggraph, in dem die Kreise die einzelnen Arbeitselemente und die Pfeile die Vorrangbeziehungen zwischen diesen darstellen.

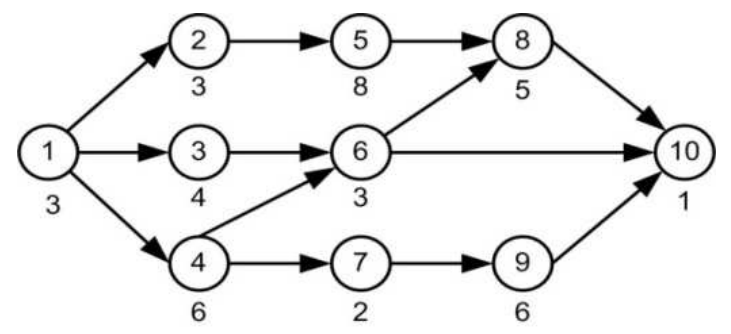

Abbildung 3: Ein Vorranggraph

Die Bearbeitungszeit des jeweiligen Elements ist unmittelbar unter dem Element angezeigt. Das Ziel ist häufig eine möglichst effiziente Aufteilung der Arbeitselemente auf die Stationen einer FPL.

Im vorliegenden Fall ist die minimale Stationenzahl zur Realisierung zu suchen. Die Produktionsrate liegt bei $\mu=1 / 11$ [Produkt/ZE. Die Lösung wird in der Abbildung 4.a dargestellt. 


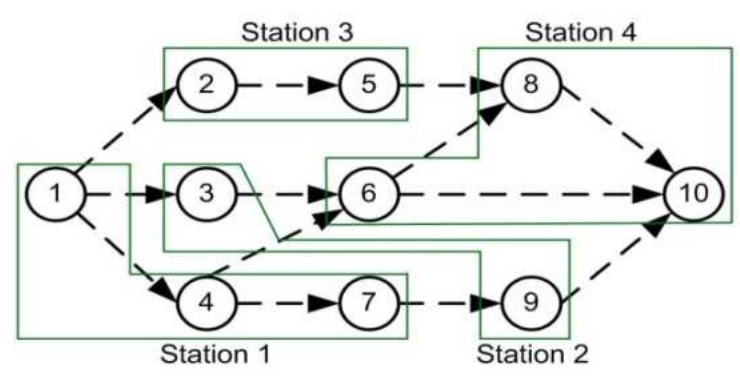

(a) 4 Stationen

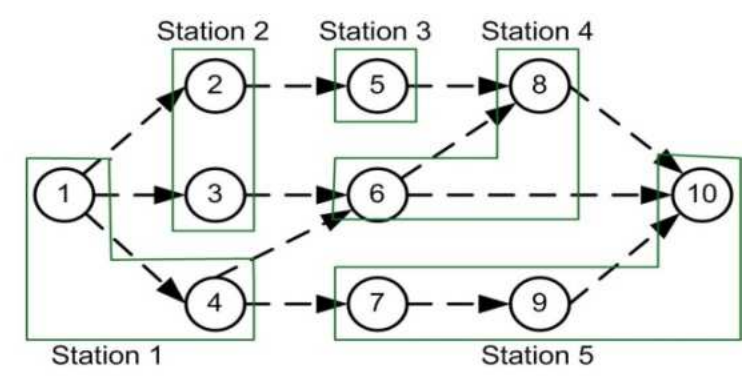

(b) 5 Stationen

Abbildung 4: Verteilung der Arbeitsmenge auf 4 bzw. 5 Stationen

Die Erhöhung der Produktionsrate auf $\mu=1 / 9$ [Produkt/ZE] erfordert eine Reduzierung der maximalen Bearbeitungszeit der Station $T_{\max }$ auf $9 \mathrm{~s}$. Das ist nicht ohne die Steigerung der Stationsanzahl $\mathrm{N}$ erfüllbar, da immer gilt:

$\mathrm{T}_{\max } \cdot \mathrm{N} \geq \sum \mathrm{t} ; \quad \sum \mathrm{t}$ : Die Summe der Arbeitszeiten aller Arbeitselemente

Der Annahme, dass die Bearbeitungsdauer eines Arbeitselements konstant ist, widersprechen Erfahrungen aus der Praxis: Bei manueller Arbeit können die Bearbeitungszeiten schwanken, da sie beispielsweise von der Motivation, Fähigkeit, Präzision etc. abhängig sind (vgl. [Boy05]). Demzufolge unterliegen die Stationen im zu untersuchenden Beispiel stochastischen Bearbeitungszeiten.

\subsection{Verzweigungs- und Zusammenführungselement}

Die Beziehungen zwischen den Einkunftsarten der Erzeugnisse vor und nach den Verzweigungselementen sind in Abbildung 5 dargestellt (vgl. [Gud04], [BGDS06]).

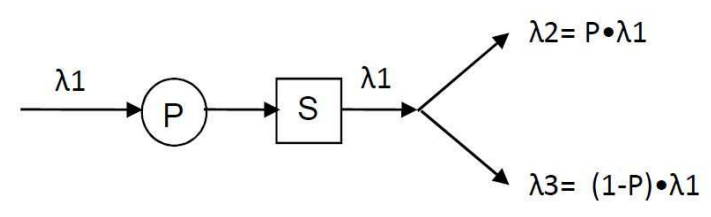

(a) Verzweigung

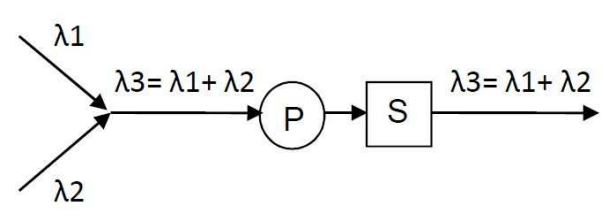

(b) Zusammenführung

Abbildung 5: Berechnung der Ankunftsrate für Verzweigung und Zusammenführung

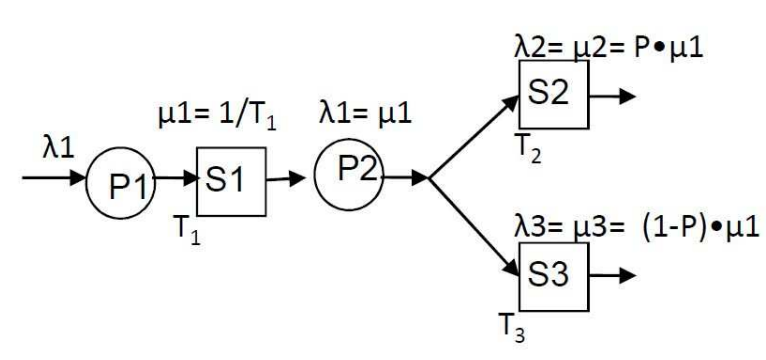

(a) Verzweigung

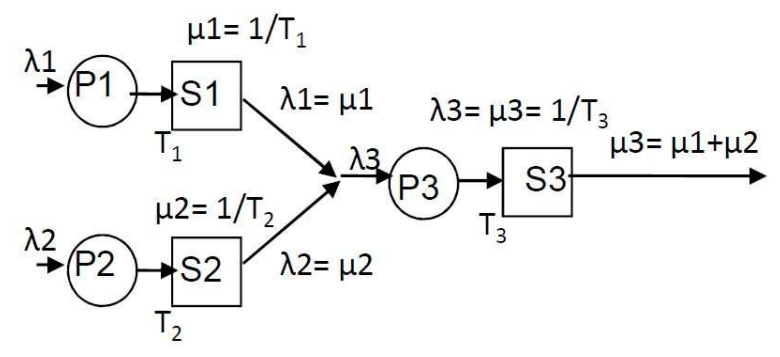

(b) Zusammenführung

Abbildung 6: Ankunftsraten und Bearbeitungsraten im idealen Fall (keine Leistungsverluste)

Bei FPS lassen sich die Verzweigung und Zusammenführung (im idealen Fall) wie in Abbildung 6 darstellen. Dabei wurde angenommen, dass die Ankunftsraten den Bearbeitungsraten gleichen. Die Gleichungen von Abbildung 5 werden in der 
Abbildung 6 erneut dargestellt. Die Bearbeitungsrate $\mu$ stellt die mittlere Anzahl der in der Station pro Zeiteinheit bearbeiteten Erzeugnisse dar und gleicht dem Kehrwert der Bearbeitungszeit dieser Station.

$\mu=\frac{1}{\mathrm{~T}}$

Verzweigungsgleichung: $\quad \frac{1}{\mathrm{~T}_{2}}=\mathrm{p} \cdot \frac{1}{\mathrm{~T}_{1}} ; \quad \frac{1}{\mathrm{~T}_{3}}=(1-\mathrm{p}) \cdot \frac{1}{\mathrm{~T}_{1}}$

Zusammenführungsgleichung: $\quad \frac{1}{\mathrm{~T}_{1}}+\frac{1}{\mathrm{~T}_{2}}=\frac{1}{\mathrm{~T}_{3}}$

Ersetzt man die Kehrwerte der Bearbeitungszeiten in den Formeln mit QSF, so ergeben sich daraus die Formeln zur Berechnung der QSF von Rohren aus Abbildung 2.

Die letzten Gleichungen dienen zur Berechnung der Soll-Bearbeitungszeiten von Stationen in einem FPS.

\subsection{Algorithmus}

In diesem Abschnitt wird eine allgemeine Vorgehensweise entwickelt, die die methodischen Schritte darstellt, welche zur Verbesserung der Konfiguration von FPS in der Planungsphase oder zur Neuplanung von existierenden Systemen, z.B. FPS mit NBS, unterstützen. Diese Schritte lassen sich wie folgt zusammenfassen:

1. Zerlegung der Struktur in Segmente: Betrachtet man die Struktur in der Abbildung 7, so lässt sie sich, je nach der erwarteten Stations- und Pufferauslastung, in vier Segmente einteilen. Die Stationen und Puffer in jedem Segment haben ähnliche Bearbeitungszeitanteile (arbeitend, blockiert, wartend). Das erste Segment umfasst alle Komponenten in der Hauptlinie, die vor der Einschleusung vorhanden sind. Das zweite Segment beinhaltet die Engpassstationen und die Puffer dazwischen. Das dritte Segment umfasst die Station S9 und Puffer P8. Das Segment 4 besteht aus allen Komponenten, die in der Nebenlinie liegen.

2. Berechnung der optimalen Bearbeitungszeiten der Stationen im jeweiligen Segment $T_{\text {bop }}$. Da jedes Segment selbst wiederum als FPL gelten kann, in der die mittleren Bearbeitungszeiten von Stationen gleich sein sollten, ist es ausreichend die Bearbeitungszeit der ersten oder letzten Station im Segment zu berechnen ${ }^{3}$.

3. Versuch, die Anzahl von Stationen im untersuchten Segment zu ändern. Das Ziel dafür ist die Erreichung der optimalen berechneten Bearbeitungszeiten $T_{\text {bop }}$. Im Fall einer Neuplanung sind die folgenden Regeln zu berücksichtigen:

- Ist die untersuchte Bearbeitungszeit des Segments $T$ kleiner als $T_{\text {bop }}$, so müsste die Stationenzahl im Segment verringert werden. Falls die Summe der Bearbeitungszeiten im Segment $\sum T$ kleiner als $T_{\text {bop }}$ ist, sind die Stationen im Segment in einer Station zu kombinieren, ihre optimale Bearbeitungszeit $T_{o p}$ gleicht dem Wert $\Sigma T$.

\footnotetext{
${ }^{3}$ Zur Vereinfachung wird der Begriff „Bearbeitungszeit des Segments“ verwendet.
} 
- Ist $\mathrm{T}>\mathrm{T}_{\text {bop }}$, so müsste die Stationenzahl im Segment vergrößert ${ }^{4}$ werden, um die Stromgeschwindigkeit am Ende des Segments zu erhöhen.

4. Je nach der Situation werden die folgenden Optimierungsmöglichkeiten untersucht, wie z.B. Änderung der Beförderungsmethode zwischen den Stationen und engpassorientierte Steuerung.

\section{Beispiel}

Um die Verbesserungsschritte anhand eines Beispiels zu erläutern, wird die in Abbildung 1 dargestellte Struktur um zusätzliche Komponenten erweitert und mit Hilfe einer Simulationsstudie untersucht (Plant Simulation). Die neue Struktur wird in vier Segmente zerlegt und in Abbildung 7 dargestellt.

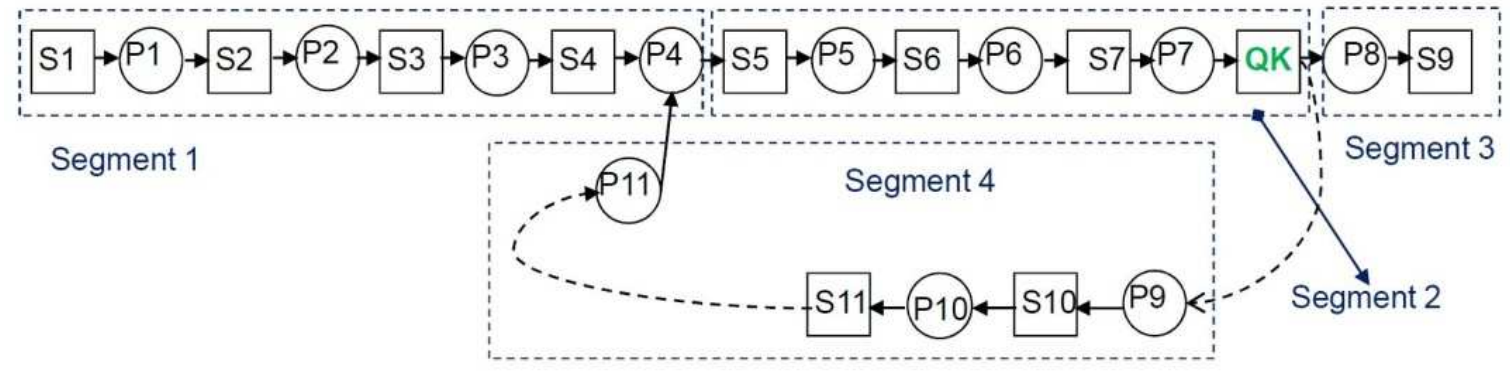

Abbildung 7: Die untersuchte Struktur

Zur Modellierung dieser Struktur werden die folgenden Annahmen getroffen:

- Die Bearbeitungszeiten der Stationen (inklusive der QK) sind gleich und normal verteilt, so dass der Mittelwert ${ }^{5} \mathrm{~T}=20 \mathrm{~s}$, die Standardabweichung $\sigma_{\mathrm{t}}=5 \mathrm{~s}$ und die untere bzw. obere Schranke 15s bzw. 25s sind. Diese Kombination aus Zeitwerten wird im Folgenden in der Form „(20, 5, 15, 25)“ dargestellt.

- Die Stationsverfügbarkeiten sind 100\%. Die Stationen sind niemals gestört.

- Der Anteil fehlerhafter Erzeugnisse ist 10\% und der perfekten Erzeugnisse ist $90 \%$.

- Die Pufferkapazitäten sind 100 Plätze für alle Puffer. Sie werden groß angenommen, damit die voraussichtlich erreichten Durchsätze nicht von den Puffergrößen beeinflusst werden.

- Die Untersuchungsdauer ist 5 Tage. Das heißt 120 Stunden.

- Die Erzeugnisse werden auf Werkstückträgern transportiert, die sich auf Rollenbahnen bewegen. Jeder Werkstückträger hat einen einzigen Platz für ein Erzeugnis.

Die Ergebnisse des Simulationsexperiments werden in der Tabelle 1 aufgeführt.

\footnotetext{
${ }^{4}$ Es ist davon auszugehen, dass sich die Arbeitsmenge auf eine größere Zahl von Stationen verteilen, d.h., den Stationen sind jeweils meist mehrere Arbeitsgänge zugeordnet. Außerdem ist diese neue Verteilung der Arbeitsmenge technologisch möglich.

${ }^{5}$ Die mittlere Bearbeitungszeit wird in diesem Beitrag auch als Bearbeitungszeit benannt.
} 


\begin{tabular}{|l|l|l|l|l|}
\hline & S4 & QK $^{6}$ & S9 & S10, S11 \\
\hline Produktmenge (Erzeugnisse) & 19538 & 21508 & 19369 & 2133 \\
\hline arbeitend (\%) & 90,48 & 99,67 & 89,84 & $9,87-9,88$ \\
\hline wartend (\%) & 0,12 & 0,33 & 10,16 & 90,12 \\
\hline blockiert (\%) & 9,39 & 0 & 0 & 0 \\
\hline
\end{tabular}

Tabelle 1: Die Ausgangssituation

Der Auslastungsgrad der Station QK, arbeitend in der Tabelle 1, ist der Höchste mit $99,67 \%$, während sie sich an Station S4 auf 90,48 \% bzw. 89,84\% an S9 reduziert. Die Ursachen dafür veranschaulicht die Tabelle 1, dass an die Station S4 Blockierung $(9,39 \%)$ und an Station S9 Wartezeiten $(10,16 \%)$ auftreten. Das führt zur Tatsache, dass sich der Durchsatz am Ende der Linie auf fast 10\% reduziert.

Je größer die Anzahl der Komponenten im Segment 1 bzw. 3 ist, desto geringer ist die Bedeutung der Funktion von Nachbearbeitungsschleifen, da die Anzahl der gestörten (blockiert und/oder gewartet) Stationen groß sind. Um den Engpass zu beheben, werden die Verbesserungsschritte in jedem Segment durchgeführt.

\subsection{Optimierung des Segments 4}

Zur Ermittlung der „berechneten optimalen“ Bearbeitungszeit $\mathrm{T}_{\text {4bop }}$ der nachzubearbeitenden Erzeugnisse im Segment 4, wird die Verzweigungsformel angewandt:

$\frac{1}{\mathrm{~T}_{4 \mathrm{bop}}}=\mathrm{P} \frac{1}{\mathrm{~T}_{1}} \rightarrow \quad \frac{1}{\mathrm{~T}_{4 \mathrm{bop}}}=0,1 \frac{1}{20}=\frac{1}{200} \rightarrow \quad \mathrm{T}_{4 \mathrm{bop}}=200 \mathrm{~s}$

Im Vergleich zur untersuchten Bearbeitungszeit $\mathrm{T}_{4}$ ist erkennbar, dass dieser große Unterschied zwischen den beiden Werten $T_{4 b o p}$ und $T_{4}$ zu erheblichen Wartezeiten im Segment 4 führt. Um der Unterschied zwischen $T_{4}$ und $T_{4 b o p} z u$ eliminieren, würde der Wert $T_{4}$ auf $T_{4 b o p}$ gesteigert. Das ist realisierbar, wenn die Summe der Bearbeitungszeiten der Stationen $\Sigma T_{4}$ als ein Wert aus den Produkten der $T_{4 b o p}$ gilt. Je geringer der Unterschied zwischen $\mathrm{T}_{4}$ und $\mathrm{T}_{4 \mathrm{bop}}$ ist, desto geringer sind die Verlustzeiten im Segment 4.

$\sum T_{4}$ ist geringer als $T_{4 b o p}$, deshalb muss nur eine Station im Segment 4 ausgelegt werden. Es wird davon ausgegangen, dass die beiden Stationen S10 und S11 in eine kombinierte Station $\mathrm{K}$ zusammengestellt werden (siehe Abbildung 8). Ihre mittlere Bearbeitungszeit $T_{40 p}$ gleicht der Summe der ursprünglichen Bearbeitungszeiten und ihre Standardabweichung entspricht der Quadratwurzel aus der Summe der ursprünglichen Varianzen (vgl. [Zoc02]).

$$
\mathrm{T}_{4 \mathrm{op}}=\sum \mathrm{T}_{4}=40 \mathrm{~s} ; \quad \sigma_{\mathrm{k}}=\sqrt{\sigma_{\mathrm{s} 7}^{2}+\sigma_{\mathrm{s} 8}^{2}}=\sqrt{25+25}
$$

Im nächsten Schritt ist das Transportprinzip zu überprüfen. Ist die Benutzung von Paletten zum Transportieren von Erzeugnissen von der QK zur Station K und/oder

\footnotetext{
${ }^{6}$ Die Angaben der letzten Station in jedem Segment sind in dieser Tabelle gezeigt. Die Station „QK“ ist identisch mit den Stationen S5, S6, S7 und befindet sich am Ende des Segments 2.
} 
von $\mathrm{K}$ zum Puffer P4 günstiger? Wenn ja, ist die Anzahl von Erzeugnissen pro Palette wirtschaftlich je nach der Situation zu ermitteln. Man geht davon aus, dass die Palettenplätze ${ }^{7}$ in diesem Beispiel 5 sind. Die neuen Daten der Station $\mathrm{K}$ lassen sich wie $(200,15.81,186,216)$ zusammenfassen. Die Abbildung 8 stellt die neue Struktur dar.

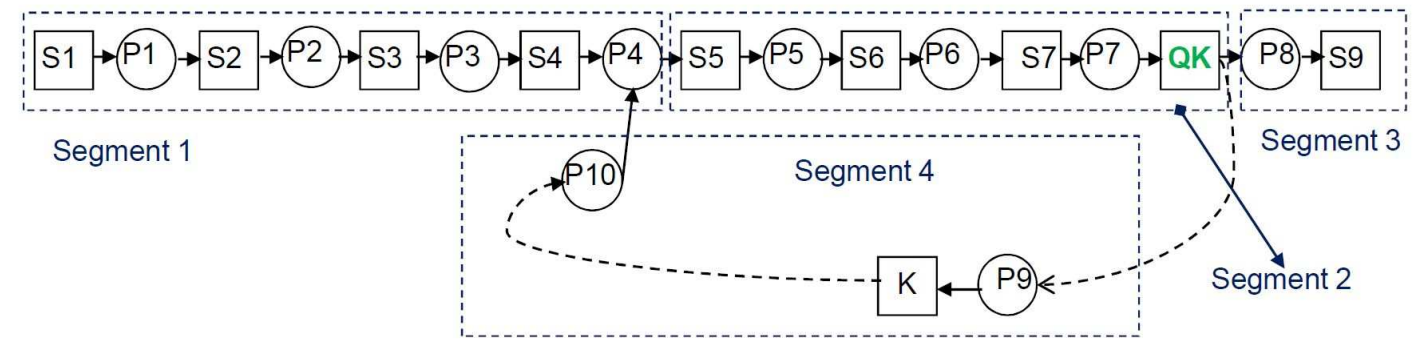

Abbildung 8: Entwicklung des Segments 4

Für die Förderung von Paletten können intelligente und autonome Funktionsmodule für den Objekttransport benutzt werden (vgl. [HSFB09]).

Hat die Reduzierung von Durchlaufzeit im Modell erste Priorität und sind die zu produzierenden Erzeugnisse teuer genug, dass sie aus wirtschaftlichen Gründen nicht vernichtet werden dürfen, muss die Struktur der Anlage erneut geplant werden (vgl. Abbildung 9.a). Nach der Implementierung der in diesem Abschnitt zur Entwicklung des Segments 4 gleichen Schritte entsteht eine neue Struktur, die in Abbildung $9 . b^{8}$ dargestellt wird.

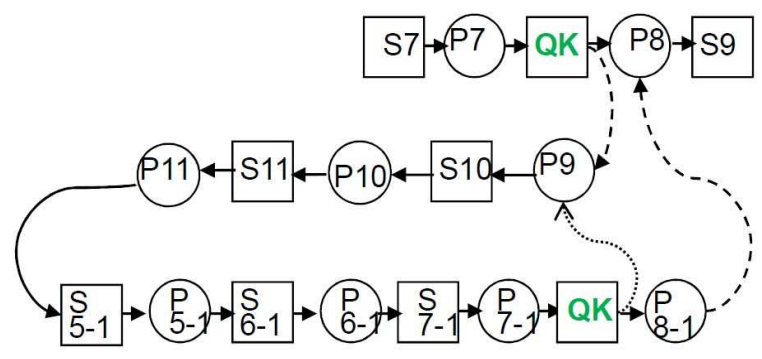

(a) untersuchte Struktur

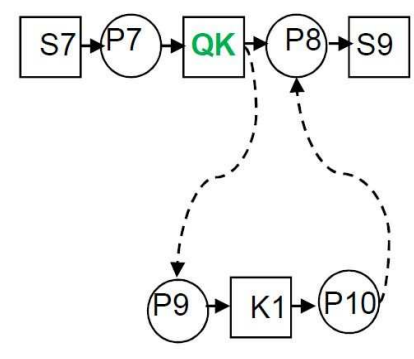

(b) optimierte Struktur

Abbildung 9: Weitere Potentiale zur Entwicklung des Segments 4

\subsection{Optimierung des Segments 2}

Eine effektive Entwicklung im Segment 2 muss in der Lage sein, den Engpass in der Hauptlinie zu verhindern. Das kann aber nicht realisiert werden, ohne die Bearbeitungszeit $T_{2}$ zu reduzieren bzw. die Stationsanzahl in Segment 2 zu erhöhen. Unter der Annahme, dass die Bearbeitungszeiten von Stationen im Segmenten 1 stabil sind, kann man $\mathrm{T}_{\text {2bop }}$ und $\mathrm{T}_{4 \mathrm{bop}}$ nach Zuführungs- und Verzweigungsgleichungen berechnen:

7 Damit die Bearbeitungszeit von Erzeugnissen, die auf einer Palette liegen, an Station K den Zeitwert T4bop nicht überschreiten, müssen $5(200 \mathrm{~s} / 40 \mathrm{~s}=5)$ Erzeugnisse auf jede Palette geladen werden.

${ }^{8} \mathrm{Je}$ nach der Situation kann die Nebenlinie nicht nur die Station K1 sondern auch QK beinhalten. 


$$
\begin{array}{r}
\frac{1}{\mathrm{~T}_{1}}+\frac{1}{\mathrm{~T}_{4 \mathrm{bop}}}=\frac{1}{\mathrm{~T}_{2 \mathrm{bop}}} \\
\left.\left.\begin{array}{r}
\frac{1}{\mathrm{~T}_{4 \mathrm{bop}}}=0,1 \frac{1}{\mathrm{~T}_{2 \mathrm{bop}}}
\end{array}\right\} \rightarrow \begin{array}{r}
\frac{1}{20}+\frac{1}{\mathrm{~T}_{4 \mathrm{bop}}}=\frac{1}{\mathrm{~T}_{2 \mathrm{bop}}} \\
=\frac{1}{\mathrm{~T}_{4 \text { bop }}}=\frac{0,1}{\mathrm{~T}_{2 \mathrm{bop}}}
\end{array}\right\} \rightarrow \frac{1}{20}+\frac{0,1}{\mathrm{~T}_{2 \text { bop }}} \\
\frac{1}{\mathrm{~T}_{2 \text { bop }}} \rightarrow \frac{0.9}{\mathrm{~T}_{2 \text { bop }}}=\frac{1}{20} \rightarrow \mathrm{T}_{2 \text { bop }}=20 \cdot 0,9=18 \mathrm{~s}
\end{array}
$$

Die Lösungen sind: $T_{2 b o p}=18 s, T_{4 b o p}=180 \mathrm{~s}$. Der berechnete Wert $T_{2 b o p}$ ist kleiner als $\mathrm{T}_{2}$, deshalb gilt dieser Wert meist als die optimale mittlere Bearbeitungszeit der Stationen $T_{\text {op }}$, aber natürlich nur, wenn es technologisch zulässig ist. Man geht davon aus, dass die Arbeitselemente ${ }^{9}$ erneut auf vier statt drei Stationen verteilt werden können. Die Abbildung 9 zeigt die zu addierende Station $Z$ und Puffer $P_{z}$.

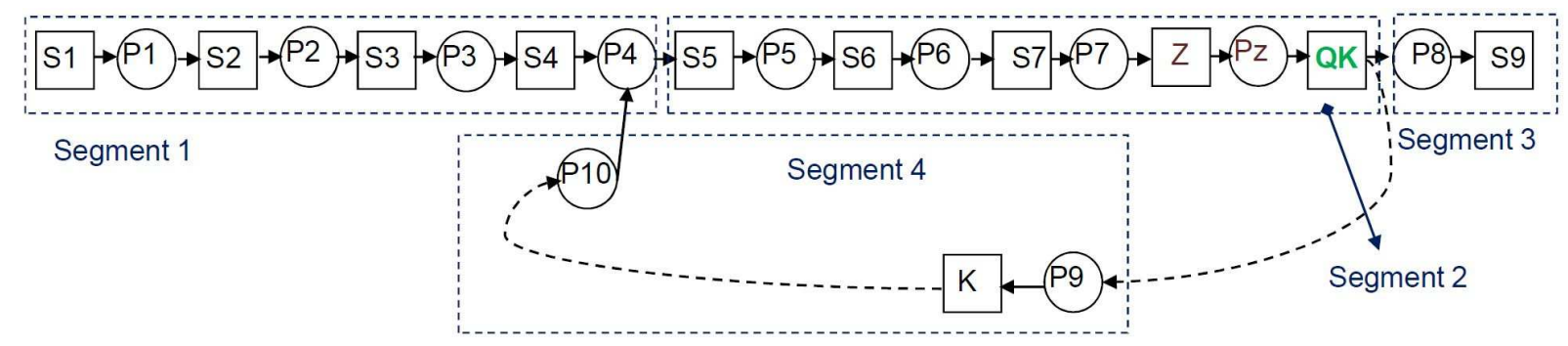

Abbildung 10: Entwicklung des Segments 2

Die in der Simulation einzufügenden Daten der Bearbeitungszeiten der Stationen im Segment 2 bzw. 4 sind zu berechnen. Für Segment 2 gilt $(18,4.56,13,23)$ und für Segment 4 gilt $(200,15.81,186,216)^{10}$. Unter Benutzung der neuen Struktur und neuen Bearbeitungszeitwerten wird ein neues Simulationsexperiment durchgeführt. Die Ergebnisse dieses Experiments werden in der Tabelle 2 dargestellt. Es zeigt sich, dass an Station 1 keine Blockierung auftritt und das Segment 2 damit nicht mehr als Engpass wirkt. Das bedeutet, die Bearbeitungszeiten der Segmente sind ausgeglichen, wodurch die Produktionsgeschwindigkeit im Engpasssegment erhöht wird.

\begin{tabular}{|l|l|l|l|l|}
\hline & S4 & QK & S9 & K \\
\hline Produktmenge (Erzeugnisse) & 21547 & 23848 & 21393 & $487 \cdot 5=2435$ \\
\hline arbeitend (\%) & 99,72 & 99,44 & 99,20 & 22,55 \\
\hline wartend (\%) & 0,28 & 0,56 & 0,80 & 77,45 \\
\hline blockiert (\%) & 0 & 0 & 0 & 0 \\
\hline
\end{tabular}

Tabelle 2: Gemessene Modellvariablen nach der Entwicklung des Segments 2

\footnotetext{
${ }^{9}$ An dieser Stelle wird unterstellt, dass die Bearbeitungszeit der QK nicht genau 20s sonder $\mathrm{T} \leq 18 \mathrm{~s}$ ist

${ }^{10}$ Mittlere Bearbeitungszeit jedes Erzeugnisses in der Station K ist 40s. Für 5 Erzeugnisse pro Palette ist die mittlere Bearbeitungszeit 200s. Es lässt sich auch annehmen, dass nur 4 Erzeugnisse pro Palette transportiert werden.
} 


\subsection{Optimierung der Segmente 1 und 3}

Praktisch lässt sich die Wiederholung der Arbeitsverteilung im Segment 2 nicht realisieren. In diesem Fall sind $T_{1 \text { bop }}$ bzw. $T_{3 \text { bop }} z u$ berechnen unter der Annahme, dass $T_{2}=20 \mathrm{~s}$ und $T_{4}=200$ stabil sind. Zunächst wird die Zuführungsgleichung zur Berechnung von $\mathrm{T}_{1 \mathrm{bop}}$ benutzt:

$\frac{1}{\mathrm{~T}_{1 \mathrm{bop}}}+\frac{1}{\mathrm{~T}_{4}}=\frac{1}{\mathrm{~T}_{2}} \rightarrow \frac{1}{\mathrm{~T}_{1 \mathrm{bop}}}=\frac{1}{20}-\frac{1}{200}=\frac{9}{200} \rightarrow \mathrm{T}_{1 \mathrm{bop}}=22,22 \mathrm{~s}$

$T_{1 b o p}$ ist größer als $T_{1}$, deshalb treten Blockierungen an diesen Stationen auf. Diese Blockierungen können theoretisch eliminiert, wenn die mittlere Bearbeitungszeit der Stationen $T_{1}$ auf $T_{1 b o p}$ steigen. Das erfordert, die Stationenzahl zu verkleinern. Die Summe der Bearbeitungszeit von Arbeitselementen im Segment $1 \sum T_{1}=60$ s ist größer als $T_{1 b o p}$, weshalb die erforderliche Stationsanzahl größer als eins ist.

Es ist zunächst zu überprüfen, ob $\sum T_{1}$ auf zwei Stationen mit dem Wert $\left(T_{1}=22,22 \mathrm{~s}\right)$ verteilt werden kann. Im Prinzip muss die folgende Bedingung realisiert werden: $\mathrm{T}_{\text {1bop }} \cdot \mathrm{N}_{1}{ }^{11} \geq \sum \mathrm{T}_{1} \rightarrow 22,22 \cdot 2=44,44<60$

Deshalb ist die Arbeitsmenge nicht auf zwei Stationen unter der getroffenen Annahme verteilbar. Ist $N_{1} \geq 9$, so kann die Stationenzahl "theoretisch“ verringert werden.

Da sich der Engpass in diesem Beispiel nicht verhindern lässt, verwendet man die Konzeption der engpassorientierten Steuerung zur Optimierung dieses Segments, so dass die Belegungstermine für alle Stationen im Segment 1 durch Rückwärtsterminierung bestimmt werden (vgl. [BCGS10]).

Zur Berechnung der $T_{3 b o p}$, benutzt man die Verzweigungsgleichung wie folgt:

$\frac{1}{\mathrm{~T}_{3 \text { bop }}}=\mathrm{P} \frac{1}{\mathrm{~T}_{2}} \rightarrow \frac{1}{\mathrm{~T}_{3 \text { bop }}}=0,9 \frac{1}{20} \rightarrow \mathrm{T}_{3}=22,22 \mathrm{~s}$

Da $T_{3 \text { bop }}>T_{3}$ ist, leidet die Station S9 unter Wartezeiten. Die Vergrößerung von $T_{3}$ lässt sich nicht realisieren, weil das Segment 3 nur eine Station umfasst. Analog zur Optimierung des Segments 1, werden die Belegungstermine für alle Stationen durch Vorwärtsterminierung bestimmt.

\section{$4 \quad$ Zusammenfassung}

In diesem Beitrag wurden Strategien und Vorgehensweisen zur Untersuchung und Optimierung von komplizierten vernetzten FPS dargestellt. Das Modell wurde in Segmente eingeteilt, so dass die Komponenten jedes Segments gleiche oder ähnliche Parameter und Eigenschaften haben. Zur Beschreibung des Verhaltens von Erzeugnissen vor, nach und in den Segmenten wurden Gleichungen zur Verfügung gestellt. Mit diesen Gleichungen können die idealen Bearbeitungszeiten der Stationen berechnet werden. Je näher die untersuchten Zeitwerte den berechneten Parametern angenähert werden können, desto größer ist die Reduzierung an Zeit-

\footnotetext{
${ }^{11} N_{1}$ ist die Stationenzahl im Segment 1.
} 
bzw. Leistungsverlust. Zur Durchführung dieser entwickelten Strategien wurde ein methodischer Algorithmus dargestellt.

Es ist von Bedeutung, bei welchem Segment die Optimierung beginnt. Im Fall eines FPS mit NBS ist zunächst das Nachbearbeitungssegment zu optimieren. AbschlieBend folgen die Segmente 2, 1 bzw. 3.

\section{Literatur}

[BGDS06]

[BCGS10]

[Boy05]

[Ger91]

[Gud04]

[HM03]

[Hel99]

[Hel00]

[HSFB09]

[KH04]

[Li04]

[Sch99]

[Zoc02]

Bolch, Gunter; Greiner, Stefan; de Meer, Hermann, S. Trivedi, Kishor: Queueing networks and markov chains: Modeling and performance evaluation with computer science applications. Hoboken, NJ : Wiley-Interscience, 2006.

Buzacott, John A.; Corsten, Hans; Gössinger, Ralf; Schneider, Herfried: Produktionsplanung und -steuerung. München: Oldenbourg, 2010.

Boysen, Nils: Variantenfließfertigung. Wiesbaden: Deutscher Universitäts-Verlag, 2005

Gershwin, Stanley B.: Assembly/Disassembly Systems: An efficient decomposition algorithm for tree-structured networks. IIE Transactions, Volume 23, Issue 4, 1991.

Gudehus, Timm: Logistik: Grundlagen Strategien Anwendungen. Berlin Heidelberg: Springer, 2004.

Heber, Stefan; Mehrtens, Nicole: Exact analysis of a continuous material merge system with limited buffer capacity and three stations. Boston [u.a.] : Kluwer Acad. Publ., 2003.

Helber, Stefan: Performance analysis of flow lines with nonlinear flow of material. Berlin [u.a.] : Springer, 1999.

Helber, Stefan: Approximate analysis of unreliable transfer lines with splits in the flow of material. Annals of operations research, Band 93. New York, NY: Springer Science, 2000.

Hippenmeyer, H.; Schönung, F.; Furmans, K.; Berbig, D.: KARIS - Kleinskaliges Autonomes Redundantes Intralogistiksystem. Düsseldorf : VDI-Verl., 2009.

Küpper, Hans-Ulrich; Helber Stefan: Ablauforganisation in Produktion und Logistik. Stuttgart: Schäffer-Poeschel, 2004.

$\mathrm{Li}$, Jingshan: Performance analysis of production systems with rework loops, Band 36. Philadelphia: Taylor \& Francis 2004.

Scholl, Armin: Balancing and Sequencing of Assembly lines. Heidelberg: Physica-Verlag, 1999.

Zocher, Klaus-Peter: Grundlagen der qualitätssichernden Fertigungsgestaltung und -steuerung. Vorlesungsskript Teil 4, Technische Universität IImenau, 2002. 